\title{
Twenty Years of Shock Wave Research at the Institute for Surgical Research
}

\author{
Michael Delius \\ Institute for Surgical Research, University of Munich, Germany
}

\section{Key Words}

Shock wave lithotripsy - Tissue damage from shock waves - Effects on tumors - Molecule transfer by shock waves

\section{Introduction}

Shock waves are acoustic pressure wave pulses which last only a microsecond and reach a pressure of several hundred up to over 1,000 atmospheres (fig. 1). They are produced outside the body and are coupled on a relatively large area through the skin into the body, propagated in tissue and focused on a stone by means of ultrasound. The history of the Institute for Surgical Research of the University of Munich is tightly linked with the development of extracorporeal shock wave lithotripsy, the fragmentation of stones in the ureteric tract and other locations. Its former director Walter Brendel set up the Institute in the sixties and directed it till his death in 1989. He started research on the clinical application of shock waves in the mid seventies.

The following brief review starts with a remark on the historical roots of stone removal and describes the evolution of extracorporeal shock wave lithotripsy. Like any other treatment, shock waves generated side effects which stimulated researchers to take a closer look at. The idea of a causative role of cavitation emerged, a process involving the generation and movement of bubbles in a fluid. This mechanism was supported by a number of experiments. It

\section{KARGER}

Fax + 41613061234 E-Mail karger@karger.ch www. karger.com
(C) 2002 S. Karger AG, Basel

0014-312X/02/0342-0030\$18.50/0

Accessible online at:

www. karger.com/journals/esr

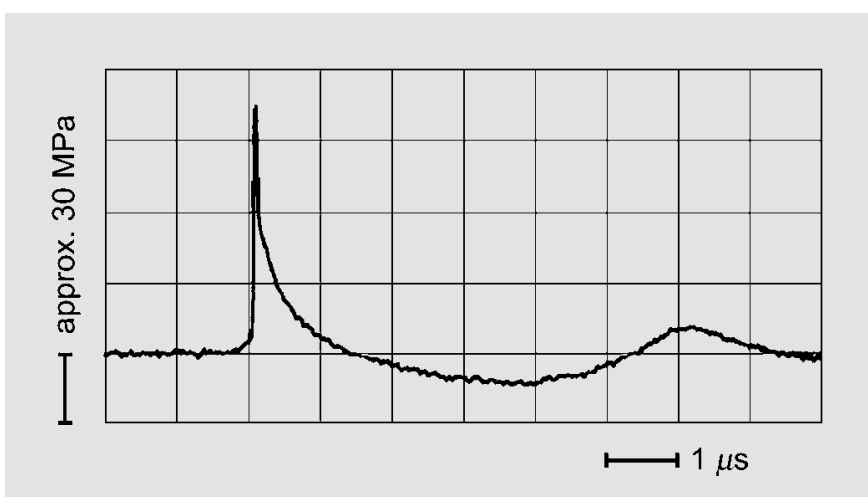

Fig. 1. Pressure registration of a shock wave of a lithotripter. The peak pressure is shown as positive upstroke on the y-axis and exceeds $90 \mathrm{MPa}$. The positive pressure wave is followed by a tensile wave which is below the baseline and is in the range of $10 \mathrm{MPa}$. The time scale on the $\mathrm{x}$-axis is in $\mu$ s. The reading was made with a fibre-optic probe hydrophone which is today the only way to provide an undistorted detection of strong tensile pressures. The principle was invented by Eisenmenger and Staudenraus [32].

remained enigmatic, on the other hand, by which physical mechanism a stone in the human body was fragmented. Only recently a convincing model has been forwarded to explain disintegration.

Lithotripsy did not remain restricted to the treatment of renal stones for long; experiments were started soon to fragment other types of stones in other locations in the body. In addition, it was attempted to apply shock waves to another key area of medical research, tumour treatment, and to examine their effect on tumour cells and on
Michael Delius MD

Institute for Surgical Research, University of Munich, Klinikum Grosshadern

D-81366 Munich (Germany)

Tel +49897095 4408, Fax +498970958897

E-Mail delius@icf.med.uni-muenchen.de 
experimental tumours in animal models. In the course of these investigations the permeability of the cell membrane was investigated in more detail. Shock waves enabled the transfer of several types of molecules into cells. It remains to be seen whether this will be another medical shock wave application beyond lithotripsy.

\section{History of Renal Lithotripsy}

For centuries people have been suffering from stones in their urinary bladders. Their removal by surgery was called lithotomy, a Greek word meaning stone cutting. The first description of the operative ways to access the bladder is said to be from Pierre Franco who lived in the Provence in Southern France in the middle of the 16th century [1]. The usual approach for lithotomy was from the perineum or the urethra and less commonly by the open abdominal route. The operation bore a certain risk since according to the records from London hospitals published in the middle of the 19th century one out of seven operations resulted in the patient's death.

In 1813 it was first proposed by Gruithuisen, a Bavarian surgeon, to grasp the stone in the urinary bladder by an instrument introduced from the urethra, drill holes into it and pulverise it. Instruments for this new strategy appeared in the following years. The first successful application of lithotripsy, a Greek word meaning stone fragmentation, was performed in 1824 in public at the French Academy in Paris. The procedure avoided surgical incisions. It replaced lithotomy in five out of six cases in the subsequent decades and lethality decreased to $7 \%$ [1].

The incidence of urinary bladder stones was high in the beginning of the 19th century and has declined greatly; today it is an uncommon diagnosis. With the introduction of general anaesthesia in 1846 by Morton in Boston and of modern asepsis in the second half of the century modern surgery emerged. Interest reverted to lithotomy, the surgical removal of the stone from the affected organ. At kidney stones, stone removal could be difficult. At gallstones, lithotomy was replaced in 1882 by Langenbuch by cholecystectomy, the surgical removal of the entire gallbladder containing the stones. It remained the mainstay of gallstone therapy till today.

The idea of lithotripsy remained that small stone fragments will pass the body after mechanical breakage by its natural outgoing tracts like ureter and bile ducts without side effect. In the fifties ultrasound was reported for the first time to fragment kidney and gallstones. Initial in vitro reports sounded encouraging yet it was found out soon that these types of stones could not be really fragmented [2]. A major breakthrough was accomplished by the in vitro fragmentation of kidney stones by shock waves, single strong ultrasonic pulses with pressures of several tens of MPa and short rise time which were propagated in a water-filled cylinder [3]. A few years later stone fragmentation was achieved in vivo in an experimental animal model when an extracorporeal approach was chosen: shock waves were generated outside the body, coupled through the skin and the overlying tissue into the body, and focused on the stone. The first human extracorporeal shock wave lithotripsy of a kidney stone was performed at the Institute for Surgical Research in 1980 [4]. Extracorporeal shock wave lithotripsy had a dramatic impact on urinary tract stone therapy. Within a few years, it became world wide the standard treatment for stones in the kidney and ureter and replaced surgery almost entirely. Many millions of patients have been treated in the last two decades. The ability to shatter the stone into small fragments which are passed with the urine during the following weeks remains a major advantage over other techniques of stone removal. The risk for the patient to suffer a major complication or death is two orders of magnitude lower than surgery.

\section{Lithotripsy at Other Locations}

The success of renal lithotripsy encouraged researchers to transfer the newly acquired knowledge and use extracorporeal shock waves for gallstone fragmentation. Animal experiments to fragment calculi in the gallbladder were initiated soon [5]. When the first patients were treated initial results were good in those with single gallstones. The fragments left the gallbladder in $80 \%$ of the treatments yet it took over a year till this was achieved [6]. A problem was that only a small fraction of patients with gallbladder stones has small single stones making them eligible for lithotripsy. An even greater problem was experienced when gallbladders with large and multiple gallstones were treated by lithotripsy. The fragment passage was incomplete in many cases. This has been confirmed in a multicenter trial in which multiple treatments with shock waves were performed [7]. Furthermore, stone recurrence was relatively high in those patients whose gallbladder emptying was naturally slow. So gallstone lithotripsy shared the same fate as gallbladder lithotomy which was introduced over hundred years ago. During this operation the gallstones were removed surgically from the bladder and the gallbladder was closed by suture. 
Follow up disclosed that the vast majority of patients formed new gallstones over the following decades. Gallbladder lithotripsy is nowadays only used in selected centres. A decade ago gallbladder removal by minimal invasive surgery was introduced and became instantly the standard therapy for symptomatic gallstones.

There is another type of gallstone which is located in the bile duct system and is readily amenable to treatment by shock wave lithotripsy. Stones in the common bile duct occur often in the elderly. Shock waves are used when attempts to remove the stone mechanically have failed. The method is a real problem solver in a difficult situation. Pancreatic stones originate from chronic pancreas inflammation. A decade ago it was shown that they can be disintegrated using shock wave lithotripsy [8]. Additional mechanical manipulations via an endoscope are usually necessary. Salivary gland stones arise in a duct in the parotid or submandibular gland. Here also shock wave lithotripsy can be employed and disintegrate them in a large percentage of cases [9].

\section{Shock Wave Effect on Tissues}

Extracorporeal shock waves have to pass tissue before they reach their target and on their way to it they can cause damage. The first hint to this side effect was the occurrence of a few renal haematomas during the first renal stone treatments. Haematuria had been noted additionally in all treatments and had been attributed to microscopic lesions from stone fragments accelerated by the the acoustic waves. Renal changes by shock waves were further characterised in the subsequent years by multiple animal experiments and a common pattern of damage was recognised $[10,11]$. Shock waves induce a type of mechanical trauma which is restricted to the high pressure area along the central axis of the shock wave field. The predominant lesion is generally damage of blood vessels. In the kidney, two types of bleedings could be differentiated, diffuse bleedings and haematomas. Diffuse bleedings demonstrated a basically preserved kidney architecture at macroscopic inspection. Microscopic inspection revealed that they were caused by a diffuse exit of red blood cells from the vascular lumen of capillaries and small and larger veins into the perivascular space; defects of the walls of arteries were only rarely detected. At electron microscopic examination, multiple small lesions of the venous and capillary walls were found as exit sites of the red blood cells [12]. The other lesion type was massive bleedings - haematomas which distended sur- rounding anatomical structures. On histology, circumferential haemorrhage into the muscular layer of arteries and focal, punched-out holes through the arterial wall were confirmed as cause of haematoma formation. Another prominent microscopical finding was the formation of blood clots in medium-sized veins; they were typically associated with a severe destruction of the venous wall of the respective vessel.

As expected, the bleedings in the kidney healed like any mechanical trauma by scar formation. Scarring could be prominent and indicated loss of functional organ tissue. No long-term impairment of renal function has been documented in animal experiments.

The kidney was the organ examined most often for shock wave effects and similar changes were found in most other tissues where shock waves were applied. Lung haemorrhage was one of the earliest documented biological side effects when shock waves were administered to gallbladders, although the organ was far away from the focus and the high pressure field [5]. The lung proved to be by far the most sensitive organ to shock waves as haemorrhage was already observed in rodents after only a few acoustic pulses of less than $5 \mathrm{MPa}$ peak pressure [13] and even at $2 \mathrm{Mpa}$ [14]. This outcome of shock wave research aroused other researchers and motivated them to examine whether similar effects could be seen when acoustic parameters were utilised like the ones in general use for diagnostic ultrasound. Animal experiments demonstrated that this was indeed the case. The consequence was that the danger of inflicting lung damage to a patient during a diagnostic ultrasound examination is now denoted at the front display in all new diagnostic ultrasound machines.

Tissue damage by shock waves in other organs like the liver and gallbladder, gut and muscle appeared just as pointed out in the kidney. Two shock wave effects deserve further mention: the action on bone and on excitable tissues. As expected from the previous experiments, the predominant lesion from shock waves focused to bone was bleeding [15]. Haemorrhage occurred at the outer bone surface, i.e. the periosteum, and within the bone marrow. The bone reacted to this shock wave trauma quite unexpectedly by an intense apposition of new bone at the site of the lesion; the cortical layer became considerably thickened. The cause of the bone apposition was not examined further, an increase of bone morphogenetic protein is expected to be found. It was speculated that the apposition accelerated the bony union of pseudarthroses treated by shock waves. Non-union of fractures is one of four entities which have been treated by shock waves starting over a decade ago which might respond to shock wave therapy; 
the other three diseases are heel spur, painful shoulder and tennis elbow. Most clinical data are from uncontrolled trials and only one of them, heel spur treatment, has been approved in the United States by the EDA in autumn 2000.

The second shock wave effect was excitation of heart and nervous tissue by shock waves. Cardiac arrhythmia was noted at the first human applications of shock wave lithotripsy; the solution had been EKG-triggering. Arrhythmia could be reproduced In experimental animals, pigs were prone to it yet dogs were not. The pressure threshold was again remarkably low in the range of $1 \mathrm{MPa}$. Twitching of muscles during lithotripsy had suggested that lithotripter shock waves could possibly excite nerves and focal membrane depolarisation could have generated action potentials. Ex vivo experiments with frog sciatic nerves demonstrated that shock waves could indeed induce compound action potentials [16]. For these to be registered it was required that small, invisible gas bubbles were present in the organ bath where the nerve was immersed. This pointed to the involvement of cavitation, a process discussed in the following chapter.

\section{Cavitation and Tissue Damage}

Cavitation is defined as the movement of newly formed and pre-existing bubbles containing gas or vapour in a fluid. Shock waves generated cavitation in tissues when administered in vivo. It was detected by diagnostic ultrasound as a briefly flashing up area in the high pressure field [17]. One second after a pulse, the change had largely disappeared. It was assumed that the diagnostic ultrasound picked up small visible gas bubbles which remained after the fast bubble oscillation. When cavitation occurred in a large blood vessel, the bubbles were observed to be flushed away with the blood flow.

It was hypothesised in the eighties that cavitation might play a causative role in the generation of tissue damage. Three findings supported this concept. The first was that there was an obvious increase in tissue damage when the shock waves were given faster. During a patient treatment, 500 to over 3,000 shock waves were administered at a rate of 1-2 discharges per second. It was argued that the time spent for a stone fragmentation could be shortened from an hour to a few seconds or minutes when 100 shock waves could be given per second; this would be again a big advantage over surgery. When such a protocol was investigated experimentally more tissue damage was found [18]. The explanation was that gas bubbles gener- ated by shock waves in the body persisted long enough to be encountered by the following shock and this interaction was damaging. The idea to shorten patient treatments was abandoned definitively when further experiments from other experimenters revealed a similar result. The second finding was the effect on tissue when shock waves were given under simultaneous injection of gelatine-stabilised air microbubbles via an arterial catheter [19]. There was a dramatic increase of haemorrhages by the microbubble injection. The explanation was again that damage was caused when gas bubbles were present. The third finding to support the role of cavitation was that tissue damage was observed exactly at the sites where cavitation had occurred and where ultrasound signals had been registered [20].

Because a role of cavitation in the generation of side effects of lithotripsy seems probable, physicists and engineers have to search for strategies to prevent or diminish the occurrence of cavitation by shock waves in the body.

\section{Mechanism of Stone Fragmentation}

During the initial days of extracorporeal shock wave lithotripsy not much thought was given to the mechanism of stone fragmentation. The unquestioned concept was that shock waves acted directly similarly to their action at other materials. Stones were fragmented at the front side, the site of shock wave entry, by compressional effects, and at the exit site by the stress from the reflected wave [21].

When the importance of cavitation for the tissue effects was recognised it was deliberated whether cavitation might also play a role in stone fragmentation. Its possible impact was backed by three experimental findings. The first was that fragmentation was suppressed by excess pressure; exposure of stones at $10 \mathrm{MPa}$ static excess pressure which corresponded to the tensile wave of the shock wave blocked fragmentation. This was in accord with previous ultrasonic experiments in which the generation and movement of cavities had been suppressed this way. The second finding was that fragmentation was suppressed in highly viscous media like glycerol or polyvinylalcohol: viscosity impedes bubble movement similarly. And last, it was recognised a few years later that a stone remained nearly untouched by shock waves when fragmentation took place in a fluid-filled vessel pressurised with minimal static excess pressure [22]. A pressure as small as one atmosphere revealed a significant result.

Recently, however, a novel mechanism of stone fragmentation has been proposed from the university of Stutt- 


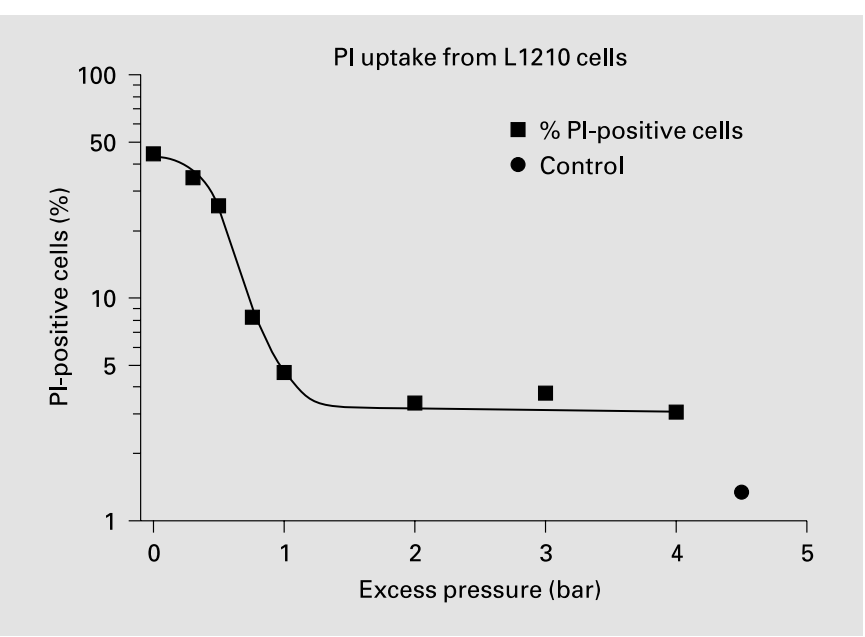

Fig. 2. Transfer of the fluorescent dye propidium iodide (PI) into L 1210 cells, a standard tumour cell line. PI does not cross the cell membrane and does not stain cells. 250 shock waves were applied to a polypropylene vial with suspended cells in medium with dissolved PI. The cells were washed to remove remnant dye and the percentage of cells with PI in the cytosol was determined in a flow cytometer and is shown on the y-axis. When the cells were exposed to shock waves at ambient pressure PI was transferred into about half of them. When the polypropylene vial was exposed to shock waves at slightly increased pressures of 1-4 bars excess pressure as indicated in the $\mathrm{x}$-axis, the percentage of positive cells decreased by $90 \%$. Co denotes the number of positive control cells that had not been exposed to shock waves.

gart [23]. It was named quasi static compression and was based on the well-known finding that human and model stones fragment in a cleavage plane perpendicular to the direction of wave propagation, i.e. directly along the midline. When a shock wave hits a stone, a pressurised region causes tensile stress within the stone in the adjoining nonpressurised areas. Maximal strains are located at the anterior and posterior stone surfaces. The fragmentation along a defined plane is easily explained this way. Compelling evidence has been presented in support of this model. When the contribution of cavitation to fragmentation is weighed against quasi static compression it seems that cavitation plays a minor role.

\section{Shock Wave Effect on Tumours}

Shock waves can be sharply focussed to an exactly defined site or a region within the body and appeared well suited for a loco-regional tumour therapy. As with any new treatment modality, the effects of shock waves on tumors stayed a focus for research for several years. The first experiments were even conducted before the appearance of tissue damage had been characterised. Shock wave application to suspended tumour cells in vitro revealed progressive, dose-dependent killing of cells. Exposure of tumours grown subcutaneously in experimental animals resulted in a retarded tumour growth. Repeated shock wave treatments were required to achieve this effect. A major mechanism of the shock wave action was probably their strong inhibitory effect on the tumor microcirculation which has been well documented [24].

Tumours are often tackled by combined therapeutic approaches, and it was therefore of interest whether shock waves enhanced the effects of other treatment modalities. Combining shock waves with hyperthermia and radiation seemed attractive because the reduction of perfusion just mentioned should help to increase the temperature in the tumor and potentiate the efficiency of free radicals: Hyperthermia was indeed superior to the shock wave treatment alone [25]. In conjunction with chemotherapy, shock waves enhanced the action of selected anticancer drugs. Dose enhancement ratios were low or absent in a number of lipophilic cytostatics [26]. The efficacy of cisplatin was among the best investigated and approximated an order of magnitude. Moreover, a previously cisplatinresistant tumor became sensitive to the drug by a shock wave treatment [27]. The effect on the resistant cells to the drug could be possibly explained by an increased permeability of the cell membrane and led to the experiments of the following chapter.

In retrospect, shock wave research on tumours had its peak in the end of the eighties, yielded limited results and has declined continuously since then. New biochemical approaches to tumour therapy and its accomplishments outweighed the progress achieved by physical techniques.

\section{Transfer of Molecules}

The enhanced effect of shock waves with cisplatin led to the hypothesis that shock waves had facilitated its entry into cells. Further investigations showed that shock waves caused a transient increase of the permeability of the cell membrane [28]. Transient cell membrane pores closed again within seconds and did not result in cell death. Exposure of cells in a fluid-filled vessel pressurised with minimal static excess pressure as shown in figure 2 reduced the number of cells with transferred propidium iodide molecules to a small fraction, suggesting that the pores were caused by fluid shear forces from cavitation. 
It was possible to transfer small molecules like the fluorescent dyes propidium iodide or calcein and also large molecules like 2-MDa dextrans into cells. In addition, solid matter like 10-nm gold particles could be transferred. Transferred molecules or particles were found in all cases in the cytosol and the cell nucleus appeared exempted. A number of investigators demonstrated the potency of transfer on biochemical cell functions. As to nucleic acid transfer, plasmids of several megadaltons size encoding standard reporter enzymes like beta galactosidase or luciferase, belonged to the large molecules which could be transduced effectively [29]. On the other hand was the synthesis of the cytokine tumour necrosis factor efficiently inhibited by a oligonucleotide composed of only twenty nucleotides [30]. As to the transfer of proteins, it was possible to deliver $30-\mathrm{kDa}$ plant proteins from a group called ribosome inactivating proteins. These are not toxic to cells since they have no receptor at the cell surface, yet once in the cytosol they inhibit protein synthesis efficiently and induce apoptosis. The action of several of these toxins was enhanced upon transfer by orders of magnitude as exemplified in the dose-response curve shown in figure 3. This has been extended to in vivo experiments and in these it was established that the shock wave-mediated transfer into the cells could be shown to be active in a tumour model [31].

So in summary shock waves increased transiently the permeability of the cell membrane and could be used as a vehicle to transfer different types of molecules into cells. The limits and limitations of this new technique have still to be evaluated.

\section{Outlook}

Major topics of shock wave research over the last two decades were renal lithotripsy with its side effects, cavitation as their possible cause, attempts to treat tumours and membrane permeability and molecule transfer. What is the scientific value of these achievements and is that all shock wave research can achieve? What will be the future?

Progress might be expected at lithotripsy and at transfer. At lithotripsy, the new model of stone fragmentation should have consequences for the design of lithotripters. It indicated that stone fragmentation can be achieved just as efficiently at comparably low shock wave pressures. The peak pressure of a new device should be lower than in lithotripters marketed so far and the focus broader. Preliminary data of a clinical study with a new type of litho-

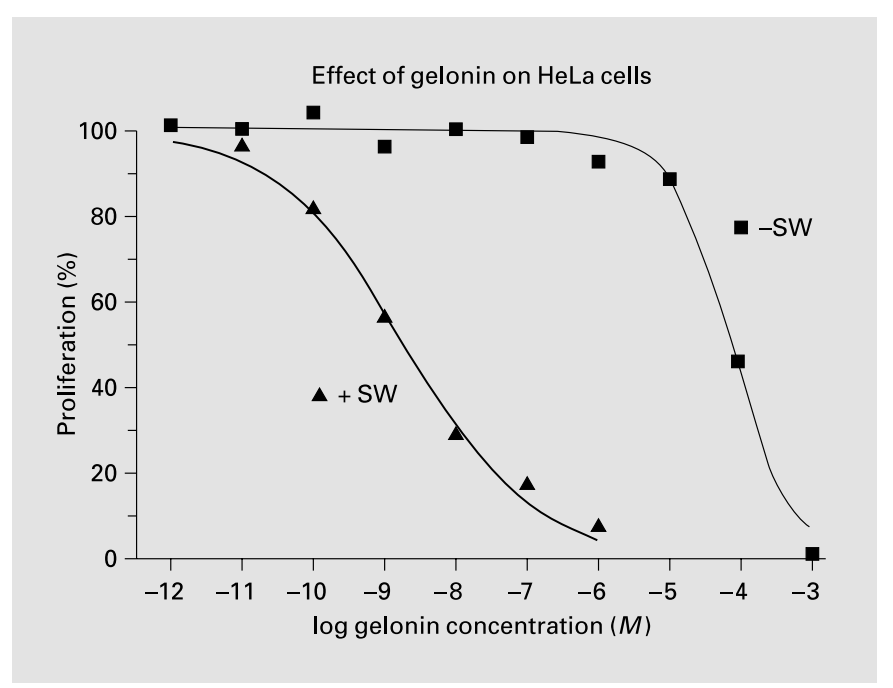

Fig. 3. Dose response curve demonstrating an enhanced action of the ribosome inactivating protein gelonin by shock waves. Suspended $\mathrm{HeLa}$ cells were incubated for $40 \mathrm{~min}$ with varying concentrations of gelonin which are shown on the $\mathrm{x}$-axis. Half of the specimens were filled into polypropylene vials and exposed to 250 shock waves in a lithotripter $(+\mathrm{SW})$, the other half served as controls which were incubated the same way with gelonin yet not treated with shock waves $(-\mathrm{SW})$. The cells were plated on microtiter plates and their proliferation was determined by MTT assay. This test is a colorimetric assay which indicates the fraction of cells which survived the treatment and could thus proliferate in culture; it is shown on the y-axis. The curve on the right depicts the proliferation of the controls. High gelonin concentrations were required to reduce proliferation. The curve on the left depicts the proliferation of shock wave-treated cells. Proliferation was reduced at low concentrations already and indicated that gelonin had been transferred into a high fraction of cells and exerted its lethal effect. Compared with the right curve there is a shift to the left of 4 log concentrations indicating the enhancement. Values are means and standard errors of the mean ( $n=5$ at each point). From [31].

tripter from China have been mentioned in summer 2001 for the first time. Lithotripsy might change considerably over the next years with a new generation of shock wave devices and one might come a bit closer to a treatment in which a lower pressure causes less pain to the patient and analgosedation is avoided. As to lithotripsy itself, the noun will only disappear when man has learnt to prevent stone formation in his body.

At molecule transfer, there are many competing physical and biochemical methods. It remains to be seen whether the increased membrane permeability can be applied in practice either in the lab or in vivo. Finding practical applications for the acoustic transfer is one of the tasks to be tackled in the future. 


\section{References}

1 Thompson H: Practical Lithotomy and Lithotripty; in Lithotomie und Lithotripsie, translated by Goldschmidt $\mathrm{H}$. Kassel, Theodor Fischer, 1882.

2 Coats EC: The application of ultrasonic energy to urinary and biliary calculi. J Urol $1956 ; 75$ : 865-874.

3 Haeusler E, Kiefer W: Anregung von Stosswellen in Flüssigkeiten durch Hochgeschwindigkeitswassertropfen. Verhandl Dtsch Physikal Ges 1971;6:786.

4 Chaussy C, Brendel W, Schmiedt E: Extracorporeally induced destruction of kidney stones by shock waves. Lancet 1980;ii:1265-1268.

5 Brendel W, Enders G. Shock waves for gallstones: Animal studies. Lancet 1983;i:1054.

6 Sauerbruch T, Delius M, Paumgartner G, Holl J, Wess O, Weber W, Hepp W, Brendel W: Fragmentation of gallstones by extracorporeal shock waves. New Engl J Med 1986;314:818822.

7 Pauletzki J, Sackmann M, Holl J, Paumgartner G: Safety and efficacy of repeated shockwave lithotripsy of gallstones with and without adjuvant bile acid therapy. Gastroenterology 1997 ; 112:1603-1609.

8 Sauerbruch T, Holl J, Sackmann M, Werner R, Wotzka R, Paumgartner G: Disintegration of a pancreatic duct stone with extracorporeal shock waves in a patient with chronic pancreatitis. Endoscopy 1987;19:207-208.

9 Iro H, Schneider H, Födra C, Waitz G, Nitsche $\mathrm{N}$, Heinritz HH, Benninger J, Ell C: Shockwave lithotripsy of salivary duct stones. Lancet 1992;339:1333-1336.

10 Delius M, Enders G, Xuan Z, Liebich H, Brendel W: Biological effects of shock waves: Kidney damage by shock waves in dogs - dose dependence. Ultrasound Med Biol 1988;14: 117-122.

11 Delius M: Medical applications and bioeffects of extracorporeal shock waves. Shock Waves 1994;4:55-72.
12 Karlsen JS, Smevik B, Hovig T: Acute morphological changes in canine kidneys following exposure to extracorporeal shock waves. Urol Res 1991;19:105-115.

13 Brendel W: Grundversuche zur Stosswellentherapie. Bundesministerium für Forschung und Technologie, Forschungsbericht $\mathrm{T}$ 86093, 1996, pp 6-34.

14 Hartman C, Child SZ, Mayer R, Schenk E, Carstensen EL: Lung damage from exposure to the fields of an electrohydraulic lithotripter. Ultrasound Med Biol 1990;16:675-679.

15 Delius M, Draenert K, Al Diek Y, Draenert Y: Biological effect of shock waves: In vivo effect of high energy pulses on rabbit bone. Ultrasound Med Biol 1995;21:1219-1225.

16 Schelling G, Delius M, Gschwender M, Grafe P, Gambihler S: Extracorporeal shock waves stimulate frog sciatic nerves indirectly via a cavitation-mediated mechanism. Biophys $\mathrm{J}$ 1994;66:133-140.

17 Delius M, Denk R, Berding C, Liebich H, Jordan M, Brendel W: Biological effects of shock waves: Cavitation by shock waves in piglet liver. Ultrasound Med Biol 1990;16:467-472.

18 Delius M, Jordan M, Eizenhoefer H, Marlinghaus E, Heine G, Liebich H, Brendel W: Biological effects of shock waves: Kidney haemorrhage by shock waves in dogs - administration rate dependence. Ultrasound Med Biol 1988; 14:689-694.

19 Prat F, Ponchon T, Berger F, Chapelon Y, Gagnon $\mathrm{P}$, Cathignol D: Hepatic lesions in the rabbit induced by acoustic cavitation. Gastroenterology 1991;100:1345-1350.

20 Delius M, Gambihler S: Sonographic imaging of extracorporeal shock wave effects in the liver and gallbladder of dogs. Digestion 1992;26:5560.

21 Forssmann B, Hepp W, Chaussy C, Eisenberger F, Wanner K: Eine Methode zur berührungsfreien Zertrümmerung von Nierensteinen durch Stosswellen. Biomed Tech 1977;22:164168.

22 Delius M: Minimal static excess pressure minimises the effect of extracorporeal shock waves on cells and reduces it on gallstones. Ultrasound Med Biol 1997;23:611-617.
23 Eisenmenger W: The mechanisms of stone fragmentation in ESWL. Ultrasound Med Biol 2001;237:615-625.

24 Gamarra F, Spelsberg F, Kuhnle G, Goetz AE: High-energy shock waves induce blood flow reduction in tumors. Cancer Res 1993;53: 1590-1595

25 Dellian M, Walenta S, Gamarra F, Kuhnle G, Mueller-Klieser W, Goetz AE: High-energy shock waves enhance hyperthermic response of tumors: Effects on blood flow, energy metabolism, and tumor growth. J Nat Cancer Inst 1994;86:287-293.

26 Gambihler S, Delius M: In-vitro interaction of lithotripter shock waves and cytotoxic drugs. Br J Cancer 1992;66:69-73.

27 Weiss N, Delius M, Gambihler S, EichholtzWirth H, Dirschedl P, Brendel W: The in-vivo effects of shock waves and cisplatin on cisplatin sensitive and resistant rodent tumors. Int $\mathrm{J}$ Cancer 1994;58:693-699.

28 Gambihler S, Delius M, Ellwart JW: Permeabilization of the plasma membrane of L1210 mouse leukemia cells using lithotripter shock waves. J Membr Biol 1994;141:267-275.

29 Lauer U, Bürgelt E, Squire Z, Messmer K, Hofschneider PH, Gregor M, Delius M: Shock wave permeabilization as new gene transfer method. Gene Therapy 1997;4:710-715.

30 Tschoep K, Hartmann G, Jox R, Thompson S, Eigler A, Krug A, Erhardt S, Adams G, Endres S, Delius M: Shock waves: A novel method for cytoplasmic delivery of antisense oligonucleotides. J Mol Med 2001;79:306-313.

31 Delius M, Adams G: Shock wave permeabilization with ribosome inactivating proteins: A new approach to tumor therapy. Can Res 1999; 59:5227-5232.

32 Staudenraus J, Eisenmenger, W: Fiber optic probe hydrophone for ultrasonic and shock wave measurements in water. Ultrasonics 1993;31:267-273 